\title{
Treatment of streptococcal pharyngitis with amoxycillin once a day
}

\author{
P Shvartzman, H Tabenkin, A Rosentzwaig, F Dolginov
}

\begin{abstract}
Objective-To evaluate treatment of group A $\beta$ haemolytic streptococcal pharyngitis with amoxycillin once daily compared with phenoxymethylpenicillin three or four times a day.

Design-Randomised controlled study of consecutive patients presenting with symptoms suggestive of group A $\beta$ haemolytic streptococcal pharyngitis in whom culture of a throat swab yielded positive results.
\end{abstract}

Setting-Five family medicine practices.

Subjects -157 patients aged over 3 years who required treatment with antibiotics.

Main outcome measures-Clinical response, bacteriological response, days at work and school lost, and compliance.

Results-During the period of the study 393 patients presented with symptoms suggesting streptococcal pharyngitis; 157 of them had throat swabs that yielded positive results on culture. Eighty two were treated with phenoxymethylpenicillin and 75 with amoxycillin. No difference was observed in the clinical response, days at work and school lost (139 days for 64 patients taking phenoxymethylpenicillin $v 100$ days for 57 patients taking amoxycillin; $p>0 \cdot 2)$, or residual positive cultures after two days $(6(7 \cdot 3 \%)$ v $3(4 \%) ; p>0 \cdot 5)$. A significant difference in the bacteriological response was found after 14 days $(5(6 \cdot 1 \%) v 0 ; p<0.04)$ with no positive cultures observed in the amoxycillin group.

Conclusion-These findings support the hypothesis that amoxycillin once daily is as effective as phenoxymethylpenicillin in the treatment of group $A$ $\beta$ haemolytic streptococcal pharyngitis.

\section{Introduction}

In general practice about a quarter of patients present with infections of the upper respiratory tract yet there is great variation in the management of these common disorders, ${ }^{12}$ among which streptococcal pharyngitis is one of the commonest.

For almost 40 years penicillin has been considered the antibiotic of choice for the treatment of group $A \beta$ haemolytic streptococcal pharyngitis. Over the years numerous clinical studies have compared benzathine or oral penicillin with other new antibiotics. ${ }^{34}$ Claims have been made that some of these agents, most recently cephalosporins, are more convenient because they can be taken once a day and may result in fewer bacteriological failures of treatment than penicillin.

Only a few studies have tried to assess compliance as it relates to frequency of administration. ${ }^{67} \mathrm{~A}$ regimen of orally administered antibiotics for streptococcal pharyngitis given once rather than several times each day would be convenient, especially for children attending school, for single parent families, and for those families in which both parents work, and may result in improved patient compliance.

Two studies have evaluated once daily oral penicillin (buffered benzylpenicillin and phenoxymethylpenicillin) and showed conflicting results. ${ }^{89}$

We evaluated once daily amoxycillin, a semisynthetic penicillin with a longer half life than those penicillins used in the previous two studies and cheaper than orally administered cephalosporin. Outcome was measured by the ability to isolate group $\mathrm{A} \beta$ haemolytic streptococcus from the pharynx 24 hours after treatment was started, the impact on clinical course, and the rate of bacteriological failure of treatment.

\section{Subjects and methods}

The study took place in five family medicine practices over six months. Consecutive patients with clinical findings suggestive of group $A$ haemolytic streptococcal pharyngitis were enrolled in the study after informed consent had been obtained. All patients were older than 3 years and were considered to require immediate treatment with antibiotics.

Patients with a history of hypersensitivity to penicillin or who had received antibiotics within the previous 72 hours, had a chronic disease, or had a personal or family history of rheumatic fever were excluded. The presence of fever, follicular exudate, enlarged lymph nodes, sore throat, dysphagia, headache, and gastrointestinal symptoms was recorded at the first visit, and afterwards patients were followed up daily by telephone until all symptoms cleared. A throat culture was obtained from each patient by vigorously swabbing the posterior pharynx and tonsils. The swab was sent in transport medium to the regional microbiology laboratory and streaked on to blood agar plate (Hy-Lab, Rehovot, Israel). The plate was then examined for the presence of $\beta$ haemolytic streptococci; a bacitracin disc (Rosco, Denmark) was placed on a subculture and incubated overnight at $37^{\circ} \mathrm{C}$. $\beta$ Haemolytic streptococci that were sensitive to bacitracin were presumptively identified as group A.

At the initial visit patients were randomly assigned to receive phenoxymethylpenicillin $250 \mathrm{mg}$ three or four times daily or amoxycillin once daily (children 50 $\mathrm{mg} / \mathrm{kg}$ and adults $750 \mathrm{mg}$ for 10 days; fig 1). All patients were asked to return in 24-48 hours for a follow up visit and a repeat throat swab. Antibiotics were discontinued in patients whose first throat culture yielded negative results, and they were not evaluated further. Others were followed up with a daily telephone call until all symptoms cleared. Patients returned for an additional throat culture 14-21 days after starting the antibiotics or if signs and symptoms recurred. Any patient who had received amoxycillin and whose throat culture yielded positive results at 2448 hours or was not improved within three days was immediately switched to a 10 day course of phenoxymethylpenicillin.

No blood samples were drawn to identify carriers and no isolation of subtypes was done. Compliance was determined by the telephone interview and during the follow up visits.
Correspondence to:

Dr Shvartzman.

BMF 1993;306:1170-2 
Bivariate analysis was performed by using $\chi^{2}$ and Fisher's exact test. Frequency distributions of the characteristics of the patients (history, symptoms, and signs) were determined and stratified by the two types of antibiotic given to assess any significant differences between the two groups. A value of $p<0.05$ was considered significant. The two groups were compared for clinical response, bacteriological response, work and school days lost, and compliance.

\section{Results}

During the study period 393 patients presented with symptoms suggesting streptococcal pharyngitis; 157 patients had a throat culture that yielded positive results and completed the $24-48$ hours' and 14 days' follow up. Eighty two patients were in the group allocated to phenoxymethylpenicillin and 75 in the amoxycillin group (see fig 1). Table I shows the

TABLE I-Characteristics of patients with group $A \beta$ haemolytic streptococcal pharyngitis. Results are numbers of patients

\begin{tabular}{lccc}
\hline & \multicolumn{2}{c}{ Drug used for treatment } & \\
\cline { 2 - 3 } Variable & $\begin{array}{c}\text { Phenoxymethylpenicillin } \\
(\mathrm{n}=82)\end{array}$ & $\begin{array}{c}\text { Amoxycillin } \\
(\mathrm{n}=75)\end{array}$ & $\begin{array}{c}\text { Total } \\
(\mathrm{n}=157)\end{array}$ \\
\hline Age (years): & & & \\
$0-4$ & 5 & 6 & 11 \\
$5-10$ & 33 & 33 & 66 \\
$11-20$ & 24 & 21 & 45 \\
$>20$ & 12 & 10 & 22 \\
Unknown & 8 & 5 & 13 \\
Women & 47 & 46 & 93 \\
Symptoms: & 64 & 58 & 112 \\
Fevert & 52 & 52 & 104 \\
Follicular exudate & & & \\
Enlarged lymph & 48 & 50 & 98 \\
nodes $\$$ & 79 & 69 & 148 \\
Sore throat $\|$ & \multicolumn{3}{l}{}
\end{tabular}

TABLE II-Number of throat cultures positive for strepcoccus according to day of treatment

\begin{tabular}{|c|c|c|c|}
\hline \multirow[b]{2}{*}{ Day } & \multicolumn{2}{|c|}{ Drug used for treatment } & \multirow[b]{2}{*}{$\begin{array}{c}\text { Total } \\
\text { No }=157\end{array}$} \\
\hline & $\begin{array}{l}\text { Phenoxymethylpenicillin } \\
(\mathrm{No}=82)\end{array}$ & $\begin{array}{l}\text { Amoxycillin } \\
(\mathrm{No}=75)\end{array}$ & \\
\hline 0 & 82 & 75 & 157 \\
\hline $2^{\star}$ & 6 & 3 & 9 \\
\hline $14 \dagger$ & 5 & 0 & 5 \\
\hline
\end{tabular}

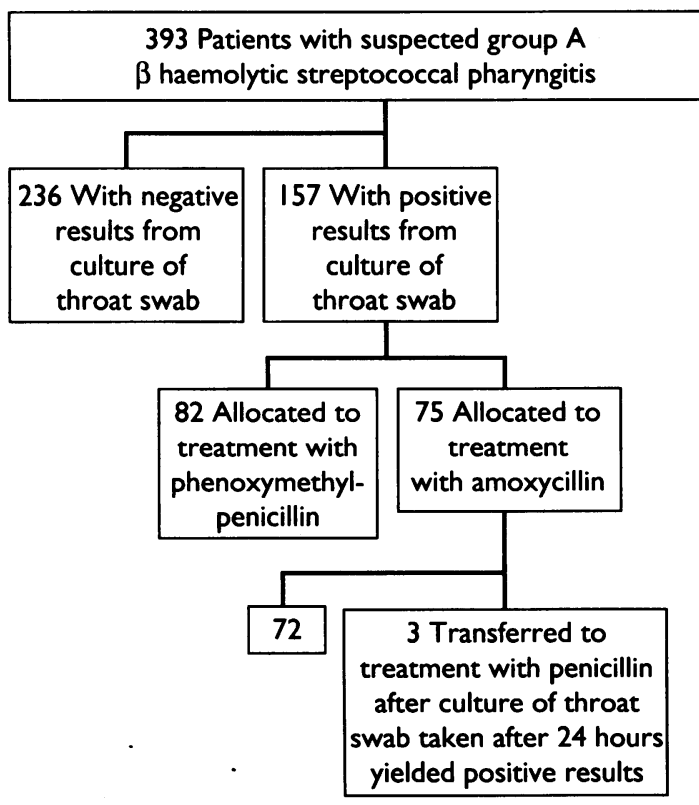

FIG 1-Flow chart of study design. All patients for whom culture of throat swabs yielded positive results were clinically followed up for 14 days or until all symptoms cleared
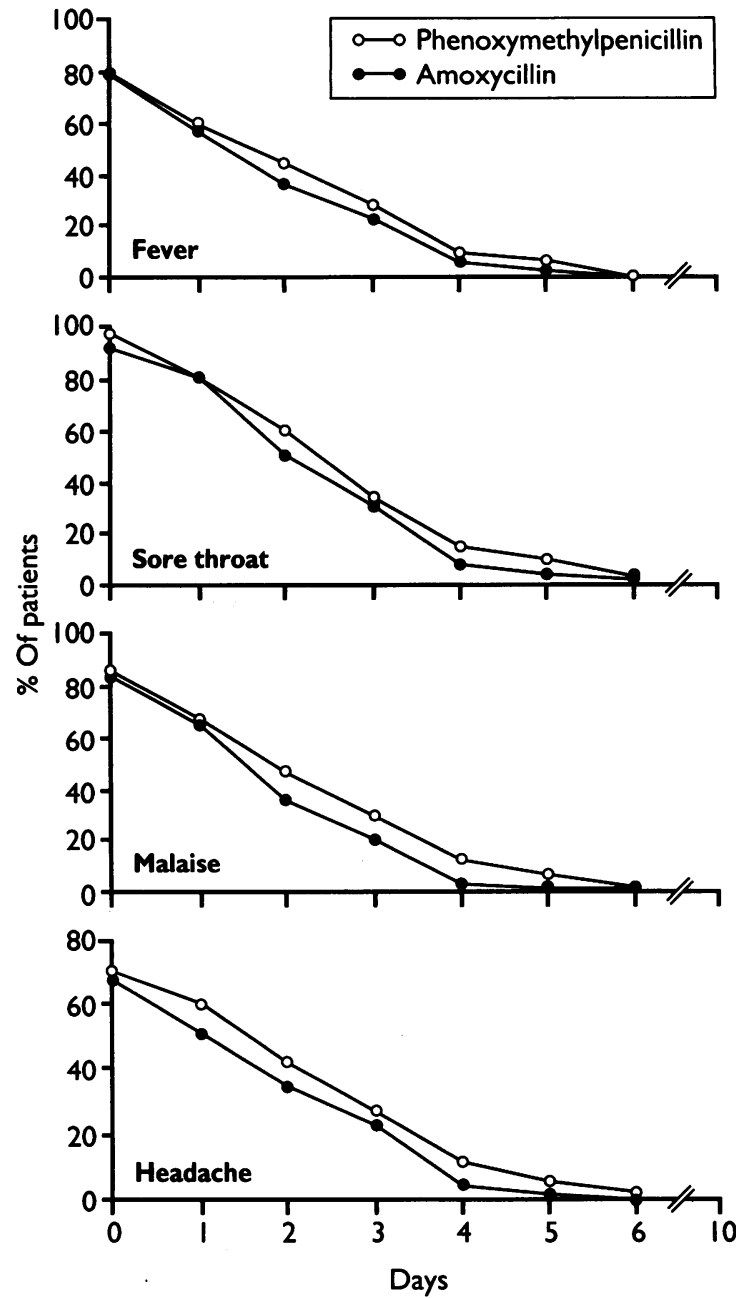

FIG 2-Effect of phenoxymethylpenicillin compared with amoxycillin once a day on four symptoms of streptococcal pharyngitis

characteristics of the study population (amoxycillin) and the control group (phenoxymethylpenicillin). No difference was observed between the groups in age distribution, sex, symptoms at time of presentation, and results of throat cultures. There was no difference in the clinical response in the two groups; figure 2 depicts, as an example, the course of fever, headache, malaise, and sore throat.

Table II shows the results of throat cultures over time. No difference was found between the two groups after 48 hours and none was noted in the days lost from school or work (fig 3).

A significantly better rate of bacteriological eradication on day 14 was observed in the amoxycillin group. There were no rashes or other important side effects with either antibiotic. The concentrations of antistreptolysin $\mathrm{O}$ and antideoxyribonuclease B were not measured nor was $M$ and $T$ typing of the throat culture isolates performed.

\section{Discussion}

The importance of adequate treatment of group A $\beta$ haemolytic streptococcal pharyngitis continued to be emphasised in medical reports as a result of recent articles documenting the continued risk of subsequent rheumatic fever..$^{10-13}$ The diagnostic and prescribing habits of general practitioners from 17 European countries for acute tonsillitis were recently assessed. The rate of prescription was high $(68 \%-100 \%)$; the lowest rate was in the Netherlands. ${ }^{14}$

A Medline search of 1965-91 found only three studies of once daily treatment with penicillin for streptococcal pharyngitis. In 1965, 50 children were 


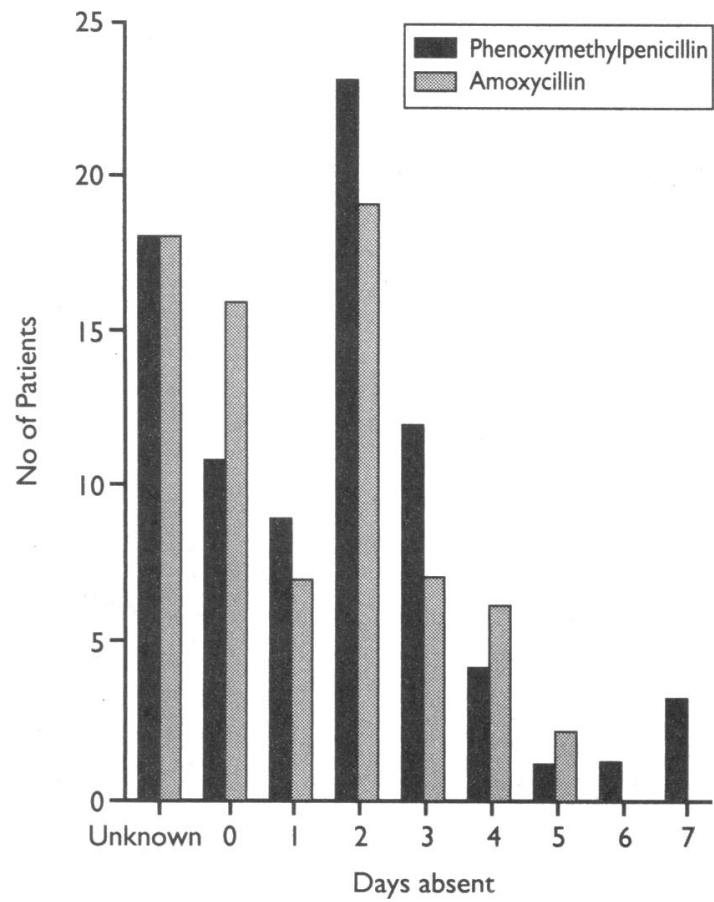

FIG 3-Distribution of days of absence from school or work of patients with streptococcal pharyngitis according to treatment with phenoxymethylpenicillin (82 patients) or amoxycillin (75 patients)

given a single dose of 800000 units of buffered benzylpenicillin potassium each day for 10 days. Forty two per cent of the patients had throat cultures that yielded positive results on follow up in the two months after completion of the study compared with $14-15 \%$ in the children of the control group. Given the fact that serotyping was not performed, bacteriological failure could not be distinguished from newly acquired disease. The authors' conclusion was that buffered benzylpenicillin was inadequate for the once daily oral treatment of streptococcal pharyngitis. ${ }^{8}$

In 1986, 196 patients with streptococcal pharyngitis were treated with once daily cefadroxial and their responses compared with those of controls receiving phenoxymethylpenicillin three times a day. A single daily dose of cefadroxil seemed to be as effective as phenoxymethylpenicillin given three times daily. The rate of bacteriological failure was only $2 \%$ in the cefadroxial group. ${ }^{5}$ In 1989, 154 patients were randomly assigned to receive phenoxymethylpenicillin once daily or three times daily for 10 days. The two regimens were comparable in their ability to eradicate the streptococcus from the upper respiratory tract in 18-24 hours and in their impact on the clinical course of the disease. The rate of bacteriological failure, however, was significantly higher in the patients taking only a single daily dose. ${ }^{9}$

Cefadroxil (an oral cephalosporin) was found to be effective when given once daily, but it is much more expensive than penicillin. The two studies which used oral penicillin showed unsatisfactory results. Amoxycillin is an oral penicillin with an excellent absorption from the gastrointestinal tract and a longer half life (30 minutes) than other oral penicillins and is considerably cheaper than the oral cephalosporins. ${ }^{15} 16$ There is no difference in cost between daily treatment with phenoxymethylpenicillin or amoxycillin. ${ }^{17}$

Amoxycillin is already regularly used for prophylaxis of endocarditis in patients with abnormal heart valves who undergo dental extraction and other procedures. It is also used to treat urinary tract infections, and in both cases a single high dose or short course is recommended.
We have shown that amoxycillin once daily (the equivalent daily dose) for 10 days is as effective in the treatment of streptococcal pharyngitis as phenoxymethylpenicillin $250 \mathrm{mg}$ three or four times a day. These regimens were comparable in their ability to eradicate the streptococcus from the upper respiratory tract and in their impact on the clinical course. They were also comparable in the number of days before patients would return to school or work.

Serotyping of isolates was not performed and concentrations of antistreptolysin $\mathrm{O}$ and antideoxyribonuclease $\mathrm{B}$ were not determined. Patients in whom the treatment had failed could not therefore be distinguished from those who were chronic carriers or had newly acquired disease. There is no reason to believe, however, that the number of carriers would be appreciably different between the two groups considering the randomisation.

The number of positive cultures on follow up, even if we could attribute all of them to bacteriological failure of treatment in both groups, were comparable and low. Diagnosis of carriers is very difficult and it is well known that early and prompt treatment may prevent a rise in titres of serum asparate transferase even in true acute infection. ${ }^{18}$

The fairly small number of patients included in the study might raise the question of type II error, but the significantly higher rate of bacteriological eradication after 14 days in the amoxycillin group makes us believe that this did not occur.

These findings support the hypothesis that amoxycillin once daily is effective in the treatment of group A $\beta$ haemolytic streptococcal pharyngitis. Furthermore, the high rate of eradication at 48 hours and the much better eradication after 14 days may suggest that there would be no difference in the non-pyogenic complications.

We thank Joe Herman, Michael Elkan, and Ron Dagan for their editorial comments.

1 De Melker RA, Kuyvenhoven MM. Management of upper respiratory tract infection in Dutch general practice. British fournal of General Practice 1991;41:504-7.

2 Raz R, Sharir R, Yalin N. Diagnosis and management of streptococcal pharyngitis. Harefuah 1989;117:390-1.

3 Dillon HC Jr. Antibiotic therapy: influence of duration, frequency, route of administration, and compliance. In: Shulman ST, ed. Pharyngitis; management in an era of declining rheumatic fever. New York: Praeger, 1984:133-51.

4 Reed BD, Huck W, Zazove P. Treatment of B-haemolytic streptococcal pharyngitis with cefaclor. 7 Fam Pract 1991;32:138-44.

pharyngitis with cefaclor. F Fam Pract 1991;32:138-44.
5 Gerber MA, Randolph MF, Chanatry J, Wright LI, Anderson LR, Kaplan EL. Once daily therapy for streptococcal pharyngitis with cefadroxil. EL. Once daily therapy

6 Ayd FJ Jr. Single daily dose of antidepressants. FAMA 1974;230:263.

7 Eisen SA, Miller DK, Woodward RS, Spitznagel E, Pnzybeck TR. The effect of prescribed daily dose frequency on patient medication compliance. Arch Intern Med 1990;150: 1881-4.

8 Breese BB, Disney FA, Talpez WB. Penicillin in streptococcal infections: tota dose and frequency of administration. Am $\mathcal{f}$ Dis Child 1965;110:125-30.

9 Gerber MA, Randolph MF, DeMeo K, Feder HM Jr, Kaplan EL. Failure of once daily penicillin $\mathrm{V}$ therapy for streptococcal pharyngitis. Am $\mathcal{f}$ Dis $C$ hild 1989;143:153-5.

10 Veasy LG, Weidmeier SE, Orsmond GS, Ruttenberg HD, Boucek MM, Roth SJ, $e t$ al. Resurgence of acute rheumatic fever in the intermountain area of the United States. N Engl f Med 1987;316:421-7.

11 Hosier DM, Craenen JM, Teske DW, Wheller JJ. Resurgence of acute rheumatic fever. $A m$ f Dis Child 1987;141:730-3.

12 Wallace MR, Garst PD, Papadimos TJ, Oldfield EC III. The return of acute rheumatic fever in young adults. ЭAMA 1989;262:2557-61.

13 Stevens DL, Tanner MH, Winship J, Swarts R, Ries KM, Schlievert PM et al. Severe group A streptococcal infections associated with a toxic shock like syndrome and scarlet fever toxin A. N Engl f Med 1989;321:1-7.

14 Touw-Otten FW, Johansen KS. Diagnosis, antibiotic treatment and outcome of acute tonsillitis: report of a WHO regional office for Europe study in 17 European countries. Fam Pract 1992;9:255-62.

15 Kitzes-Cohen R, Goldschmid A, Fostick MA. Drug formulary. Tel Aviv: Kupat Holim-Health Insurance of the General Federation of Labour in Israel, 1992:37.

16 Reynolds EF, Parsons AV, Sweetman SC. Martindale-the extra pharmacopoeia. 29th ed. London: The Pharmaceutical Press, 1989:115.

17 Combs JT. Amoxicillin therapy for strep throat. Pediatr Infect Dis $\mathcal{Y}$ 1992;11:132.

18 Georges P. Streptococcal pharyngitis: current therapy and criteria for evaluation of new agents. Clinical Infectious Diseases 1992;14(suppl):218-23. 